\title{
Performance Characterization of Nanofiltration Membranes Based on Rigid Star Amphiphiles
}

\author{
Tasuma Suzuki ${ }^{\dagger}$, Yunyi Lu $u^{\ddagger}$, Wei Zhang ${ }^{\ddagger}$, Jeffrey S. Moore ${ }^{\ddagger \$}$, Benito J. Mariñas ${ }^{*}, \S$ \\ ${ }^{\dagger}$ Department of Civil and Environmental Engineering, University of Illinois at Urbana- \\ Champaign, Urbana, Illinois 61801 \\ $\$$ Departments of Chemistry and Materials Science and Engineering, University of Illinois at \\ Urbana-Champaign, Urbana, Illinois 61801 \\ $\S$ NSF Science and Technology Center of Advanced Materials for the Purification of Water with \\ Systems, University of Illinois at Urbana-Champaign, Urbana, Illinois, 61801

\section{Supporting Information}

* corresponding author phone: (217) 333-6961; fax: (217) 333-6979: e-mail: marinas@uiuc.edu 

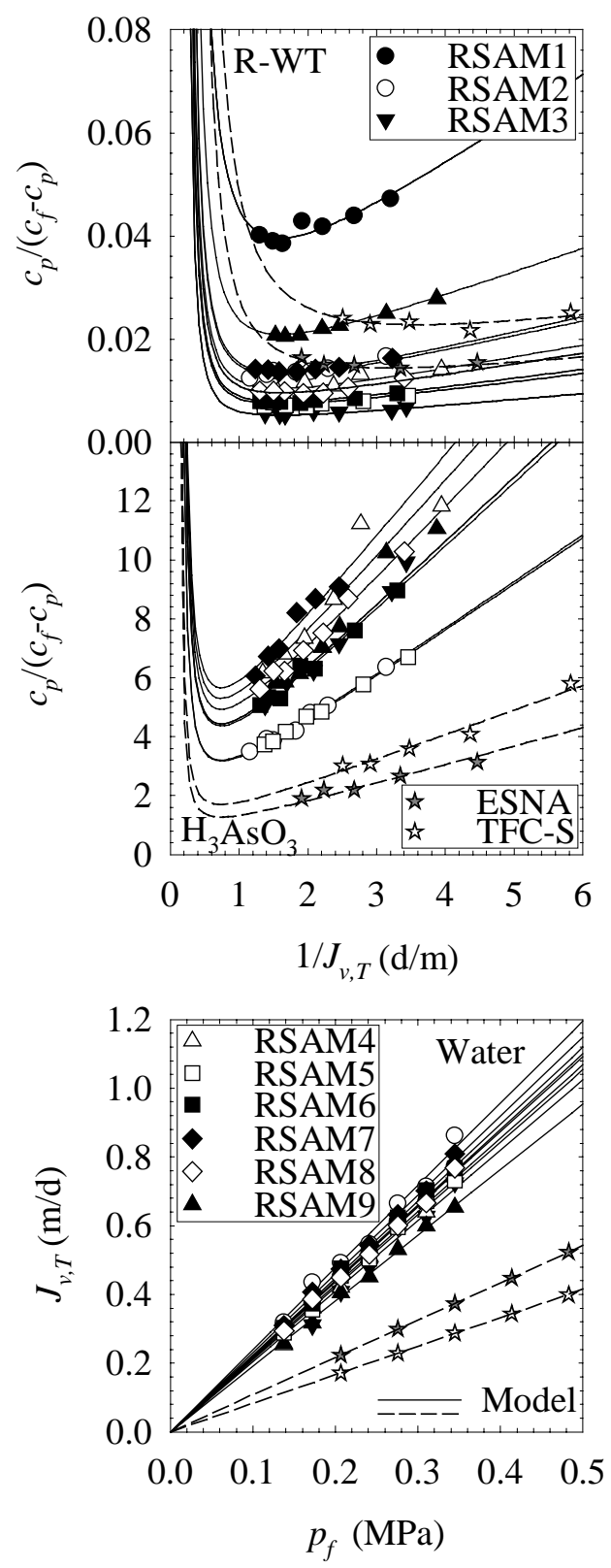

Fig. S-1. Experimental (symbols) and modeled (continuous lines) water flux and solute (R-WT and $\mathrm{H}_{3} \mathrm{AsO}_{3}$ ) permeation of RSAM-Ms (medium water permeability) made with all nine RSAs and tested at high mixing. Also shown are the experimental (star symbols) and modeled (dashed lines) water flux and solute (R-WT and $\mathrm{H}_{3} \mathrm{AsO}_{3}$ ) permeation obtained for commercial NF membranes (ESNA, TFC-S) tested under similar conditions. 

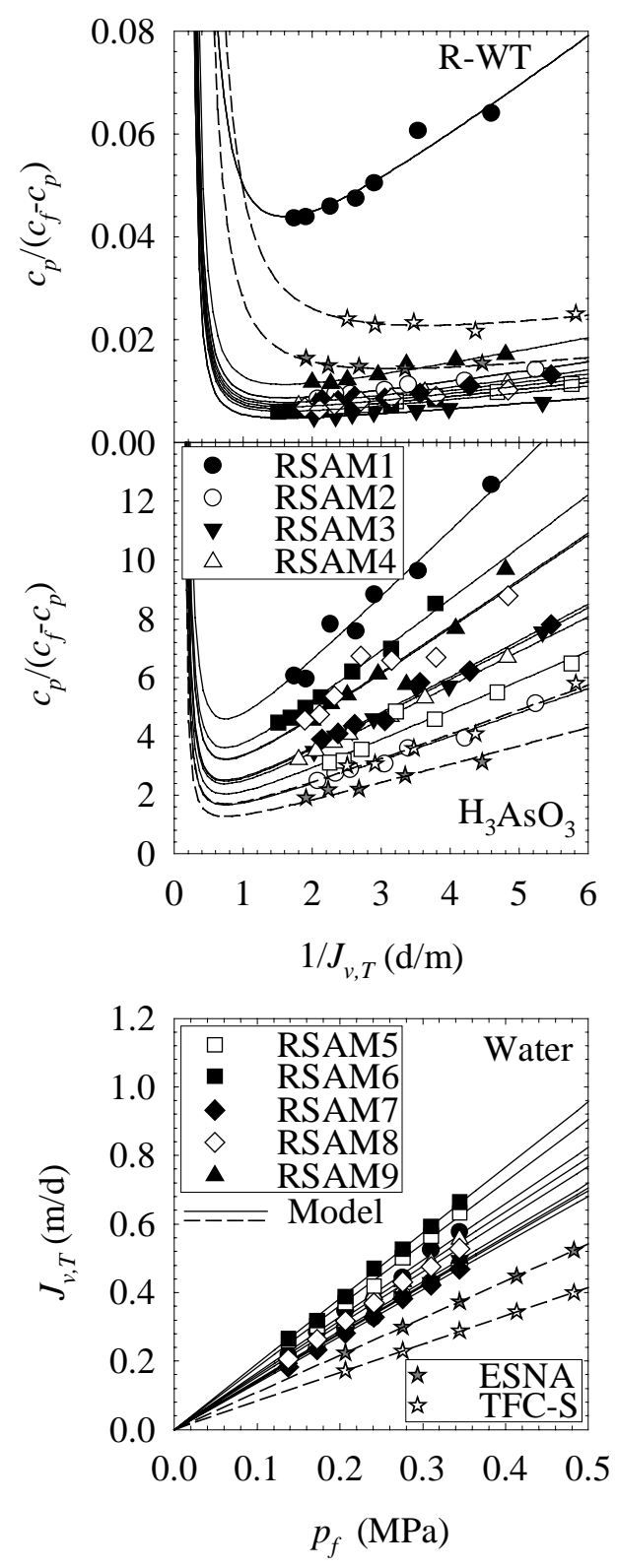

Fig. S-2. Experimental (symbols) and modeled (continuous lines) water flux and solute (R-WT and $\mathrm{H}_{3} \mathrm{AsO}_{3}$ ) permeation of RSAM-Ls (low water permeability) made with all nine RSAs and tested at high mixing. Also shown are the experimental (star symbols) and modeled (dashed lines) water flux and solute (R-WT and $\mathrm{H}_{3} \mathrm{AsO}_{3}$ ) permeation obtained for commercial NF membranes (ESNA, TFC-S) tested under similar conditions. 